\title{
The Edible and Medicinal Value of Coix lacryma-jobi and Key Cultivation Techniques for High and Stable Yield
}

\author{
Li Feng1, Yonghong Zhao², Zhuqi Zhang², Shengai Zhang², Hongxia Zhang ${ }^{3 *}$, Mingli Yu2 ${ }^{2}$, Yiwei Ma² \\ ${ }^{1}$ Shuangyashan Agricultural Broadcasting and Television School, Shuangyashan, China \\ ${ }^{2}$ Binzhou Vocational College, Binzhou, China \\ ${ }^{3}$ Bincheng District Agricultural and Rural Bureau of Binzhou, Binzhou, China \\ Email: ^wyy6677@126.com, *yumingli2002@163.com
}

How to cite this paper: Feng, L., Zhao, Y.H., Zhang, Z.Q., Zhang, S.A., Zhang, H.X., Yu, M.L. and Ma, Y.W. (2020) The Edible and Medicinal Value of Coix lacryma-jobi and Key Cultivation Techniques for High and Stable Yield. Natural Resources, 11, 569-575.

https://doi.org/10.4236/nr.2020.1112034

Received: November 16, 2020

Accepted: December 26, 2020

Published: December 29, 2020

Copyright $\odot 2020$ by author(s) and Scientific Research Publishing Inc. This work is licensed under the Creative Commons Attribution International License (CC BY 4.0).

http://creativecommons.org/licenses/by/4.0/

\section{(c) (i) Open Access}

\begin{abstract}
In this paper, it was introduced that Coix lacryma-jobi is a traditional dualuse edible and medicine crops in China, and the nutritional components and medicinal value of Coix lacryma-jobi were described. Based on the published literature at home and abroad, a variety of chemical components found in Coix lacryma jobi and their medicinal effects were reviewed. At the same time, the problems existing in the production of Coix lacryma jobi were put forward. With the improvement of people's living standards, the nutritional and health care value of Coix lacryma-jobi has been paid more and more attention. The problems of Coix lacryma-jobi in production were analyzed. Some biological characteristics of Coix lacryma-jobi, especially its hygrophyte characteristics, were re-recognized, which is very important to increase the yield of Coix lacryma jobi. In the paper, the key cultivation techniques for high and stable yield of Coix lacryma-jobi were also summarized. In order to obtain high quality, high and stable yield, we should adopt the new technical methods of "two wet, two dry and two water" in water management. At the same time, if artificial pollination is applied in the peak of flowering period, the seed setting rate and the yield will be increased.
\end{abstract}

\section{Keywords}

Artificial Pollination, Edible and Medicinal Value, High and Stable Yield, Key Cultivation Techniques, Yellow River Delta

\section{Introduction}

Coix (Coix lacryma-jobi L.), belonging to Coix in Gramineae, is an annual or

${ }^{\star}$ Hongxia Zhang and Mingli Yu are tied for first corresponding author. 
perennial herb with thick and tall stalks, commonly known as bodhi bead, sixmillet, pearl rice, medicinal corn, Coix bead etc., and the seed after hulled, is also called Coix rice, Coix seed, job's tears rice, etc. Coix lacryma-jobi is an important crop having medicine and food function with important medicinal and economic value, and it is widely distributed in tropical and subtropical regions of the world [1] [2]. In China, it is mainly planted (or wild) in Guizhou, Yunnan, Sichuan, Fujian, Taiwan, Guangdong, Guangxi, Hainan, Shandong, Shanxi, Henan, Hebei, Liaoning and other places [3] [4]. Ishiguro et al. isolated 3,5-dimethoxy-1hinden-1-one with antibacterial activity from Coix seeds and the results showed that the compound had certain effects on bacteria, yeasts and fungi. In addition, benzoxazinone compounds extracted from Coix lacryma jobi root also had antibacterial activity [5]. Coix is widely used not only in medical treatment, health care and food, but also in beauty.

Coix lacryma-jobi seeds are one of the traditional food and medicine resources in China, which can be used to make porridge, rice and various pastas for people to eat. They are sweet in taste and cold in nature, and have the effects of strengthening spleen and dampness, clearing heat and detoxification, beautifying and beautifying [3] [6]. They can also be used as drug to treat various diseases. It is recorded in the illustrated handbook of Chinese herbal plants, Coix lacryma-jobi seeds could treat pulmonary edema, wet pleurisy, chronic gastrointestinal disease, chronic ulcer, dysuria and other diseases. According to traditional Chinese medicine, Coix seeds had the function of promoting dampness and diuresis, clearing heat and removing pus, tonifying spleen, stopping diarrhea and eliminating dampness [7]. Coix lacryma-jobi is not only a traditional and ancient crop, but also a crop that is re-recognized by people today. In recent years, because Coix seeds have a special function of food therapy and health care, and the planting of Coix has very high economic benefits, therefore, the planting area of Coix lacrymajobi is expanding rapidly [1]. However, due to the lack of comprehensive understanding of the biological characteristics, the lack of in-depth study on the cultivation techniques of Coix lacryma-jobi and traditional extensive field management in production, the yield and benefit of Coix lacryma-jobi have been affected very much [8]. In this paper, some nutritional health value and biological characteristics of Coix lacryma-jobi, especially its hygrophyte characteristics, were re-recognized, and the cultivation techniques of high quality, high and stable yield were summarized in order to provide the scientific reference for the development of Coix lacryma-jobi cultivation.

\section{Nutritional Value of Coix}

The hulled seeds of Coix lacryma-jobi are called Coix rice. The nutritional value of Coix rice is very high, and the nutrition is relatively comprehensive. It ranks the first among the gramineous crops and is known as "the king of gramineous crops in the world". According to the determination by Chinese Academy of Sciences, Coix rice contains $14 \%$ protein, $5 \%$ fat, $56 \%$ carbohydrate, $3 \%$ crude 
fiber, $0.07 \%$ calcium, $0.224 \%$ phosphorus and $0.001 \%$ iron, the content of these components is higher than that of rice. In addition, Coix rice also contains vitamin B1, vitamin B2, vitamin E, niacin, dietary fiber and other nutrients [9]. Coix rice is also a kind of green food with high protein, medium fat, medium sugar and rich zinc. Many studies have shown that Coix rice not only contains a large amount of protein, but also contains many kinds of amino acids necessary for human body. Hu Jun et al. (2007) reported that Coix lacryma jobi seeds contained 8 kinds of amino acids necessary for human body, and the proportion of them is very close to the needs of human body, and they are easier to be absorbed and used by the body than that of rice [5]. Besides, Xie Jing et al. (2016) reported that the content of calcium in Coix seeds was higher than that in other grains [10]. Because Coix rice contains rich nutrients and high calorie, and it can promote metabolism and reduce gastrointestinal burden, so it is a very good tonic food for patients or weak people.

\section{Medicinal Value of Coix lacryma-jobi}

Coix lacryma-jobi is a Chinese traditional medicinal crop, which has many medical effects (e.g. diuretic, spleen, lung, dispel dampness and heat, eliminate edema, discharge pus, stop diarrhea, etc.). Early medicine studies showed that Coix seed had antipyretic, analgesic and sedative effects, and has an exciting effect on isolated heart, intestine and uterus, etc. [11]. As early as in Shennong materia medica classic, there was a clear record: "the main tendons were urgent convulsion and constricted, do not bend rheumatic arthralgia, lower vitality, take long, light body and replenish vitality" [7]. In addition, it was also said in Compendium of Materia Medica: "Coix rice porridge can remove dampness and heat, and benefit intestines and stomach. At present, the researches on the medicinal value of Coix are mainly focused on Coix seeds. Yumei Zhang et al. reported that the roots, stems, leaves and seeds of Coix lacryma-jobi could be used as medicine, specially the seed of it had higher medicinal value [2] [5]. The comprehensive research showed that Coix rice contained many active chemical components (e.g. lipids, polysaccharides, lignans, phenols and adenosine, ect). Some scholars' researches showed that Coix seed oil contained odd carbon chain fatty acids with strong anticancer activity, which had significant inhibitory effect on tumor growth. There are two kinds of sitosterol (e.g. $\beta$-sitosterol and $\gamma$-sitosterol) in Coix rice, which have high medicinal value. These special active components may be the important material basis for the anti-cancer effect of Coix rice [9]. In addition, Coix rice has the effect of preventing hyperlipidemia, hypertension, stroke and cardiovascular diseases, etc. Coix rice is also a kind of food of improving one's looks, which can keep the skin lustrous and delicate. It can eliminate acne, freckles, senile plaque, pregnancy spots and butterfly spots, which has good curative effect on hemorrhoids and rough skin [11]. In short, Coix lacryma jobi, as a traditional Chinese medicine plant in China, has both medicinal and edible functions. Therefore, it is necessary to conduct in-depth research and develop- 
ment in medicine and health food of Coix lacryma jobi.

\section{Key Techniques of Coix lacryma-jobi for High and Stable Yield}

\subsection{Cultivation Season}

The growth period of Coix lacryma-jobi is longer, generally, it is $125-180$ days. It is suitable to select varieties with a duration of more than 160 days in South China, about 150 days in North China and about 125 days in Northeast China. The sowing time is generally as follows: early maturing varieties are sown in early March, middle maturing varieties are sown from late March to early April, and late maturing varieties are sown from late April to early May. Sowing should be early rather than late. If sowing is too late, it will affect the yield and even the seeds can not mature after autumn [8] [12]. In North China, especially in the Yellow River Delta region, early maturing variety is the best choice for Coix cultivation, which should be arranged to be planted in summer autumn season. That is: sowing in late spring and early summer, harvesting in early October. In this season, there is abundant rainfall and even water logging in this region, but it just meets the characteristics of hygroscopicity of Coix lacryma-jobi, which can promote the growth and development of Coix lacryma-jobi and improve the yield. In this area, if spring sowing is adopted, it is easy to be harmed by drought, at the same time, it is easy to be harmed by dry and hot wind weather at heading stage. In this way, the pollen loses its vitality and can not be pollinated, which leads to the hollow seeds, and the yield will be reduced in light and the yield will be zero in heavy.

\subsection{Crops for Rotation}

Crop rotation arrangement should consider not only the time of succession, but also the effects of previous crop on soil nutrients, diseases and insect pests (especially soil borne diseases). Most of the medicinal plants are not resistant to continuous cropping, But Coix lacryma-jobi is a gramineous crop, and its autotoxicity is less. In general, rice, soybean and rape are the best previous crops of Coix lacryma-jobi [13] [14]. In the Yellow River Delta, Coix lacryma-jobi should be arranged to be planted in summer autumn season, which can be rotated with overwintering crops (e. g. garlic, onion, overwintering spinach and overwintering wheat, etc.). In the Yellow River Delta region, overwintering crops such as wheat, garlic and onion are usually harvested in the first ten days of June. When the wheat, garlic and onion are harvested, the land preparation will be done simply or even not, and then Coix lacryma-jobi will be sown in time. If the sowing is delayed, the seeds of Coix lacryma-jobi will not mature well.

\subsection{Land Selection}

Coix lacryma-jobi is a kind of hygrophic plant, which likes soil moisture and has strong resistance to soil moisture [12]. Therefore, we can choose low-lying and 
waterlogged plots of land, but at the same time, we should prepare good irrigation conditions to ensure that the soil moisture is sufficient in the early growth stage and flowering stage of Coix lacryma-jobi. In the early growth stage and flowering stage of Coix lacryma jobi, if the soil is short of water or drought, the yield will be reduced [15].

\subsection{Variety and Density}

Density is an important factor affecting the yield of Coix lacryma jobi. In order to obtain high yield, we must adopt reasonable planting density. Reasonable planting density depends on cultivation season, variety type and soil fertility [16]. As far as the cultivation of Coix lacryma jobi is concerned in the Yellow River Delta, the cultivation in summer autumn season is applied, it is better to select the variety of early maturing red shell Coix lacryma jobi in Liao Ning. The planting density is generally that row spacing is $25-30 \mathrm{~cm}$, the plant spacing is $10-13 \mathrm{~cm}$, and the number of seedlings is $20,000-26,000$ plants per $667 \mathrm{~m}^{2}$ [13] [17].

\subsection{Water Management}

Water management is the key to obtain stable and high yield of Coix lacryma jobi. In the past, people used to think that Coix lacryma jobi was an xerophyte, and the cultivation method of dryland was often used in production, as a result, no high yield has been obtained [18]. In recent years, with the development of Coix lacryma-jobi cultivation technology and the practice of many growers, it has been proved that Coix lacryma jobi is not a dry crop, but a hygrophic crop with strong moisture tolerance. In order to obtain high quality, high and stable yield, it is necessary to correct the misunderstanding of dryland cultivation on Coix lacryma jobi, and adopt the wet cultivation method similar to rice [4] [19]. If the Coix lacryma jobi is planted on dry land, it is necessary that sufficient irrigation conditions must be ensured. We should adopt the technical methods of "two wet, two dry and two water" in water management. That is: the soil should be kept to be moist in the early stage of growth to promote seedling growth, dry farming should be applied at jointing stage, there is water in the field at booting and heading stage, the soil should be kept to be moist at filling stage, and the soil should be kept to be dry during harvest period.

\subsection{Artificial Pollination}

Coix lacryma jobi is a monoecious and different flower spikes plant. In the same inflorescence, the male flower matures first, maturation period of which is not consistent with that of the female flower. Female flowers often need to be pollinated by the pollen from different plants. Coix lacryma jobi can be pollinated by wind pollination, but if artificial pollination is applied in the peak of flowering period, the effect of pollination would be more better [10] [15]. The specific method is that the ropes, long bamboo poles and other tools are pulled forward 
horizontally at 10 - 12 a.m. on sunny days over the plants of Coix lacryma jobi, and make the plants vibrate continuously, so as to make the pollen fly, which can increase the seed setting rate and the yield.

\section{Concluding Remarks}

In China, Coix lacryma jobi is not only a traditional medicinal and edible crop, but also an important modern medicinal plant. With the development of society and the improvement of people's living standard, the health value of Coix lacryma jobi has been paid more and more attention. Therefore, the development of plant resources of Coix lacryma jobi is imperative in the future. At present, the production of Coix lacryma jobi is mainly distributed in the southwest and southeast of China, but less in the north. The main reason is that most of the existing Coix lacryma jobi varieties are late maturing varieties with long growth period, and lack early maturing varieties with short growth period. However, the climate and environmental characteristics in north of China are very conducive to the synthesis and accumulation of the nutritional contents in Coix seeds, so it's good for the production of high-quality Coix rice. Therefore, in the future, we should strengthen the breeding of early maturing varieties with short growth period, popularize new, simple and low-cost production technology, especially vigorously popularize the mechanized sowing and mechanized harvesting technology of Coix lacryma jobi, which is the key to develop the production of Coix lacryma jobi in north and even northeast of China. At the same time, it is an urgent problem to explore the cultivation model and key technology to increase the content of medicinal components in Coix seeds.

\section{Acknowledgements}

This paper is funded and supported by Modern Agronomic Skill Master studio of Binzhou and Circular Agriculture Master Studio of Binzhou Vocational College.

\section{Conflicts of Interest}

The authors declare no conflicts of interest regarding the publication of this paper.

\section{References}

[1] Zhang, Y.B., He, X.H., Liu, F.Z., Meng, Q.Y., Liu, R. and Shen, G. (2018) Screening and Analysis of Germplasm Resources of Wild Coix lacryma-jobi L. with High Content of Coixin. Mecical Plant, 2, 66-68.

[2] Xie, J., Liu, L.Z., Lu, M.M. and Wang, Y. (2016) Research Progress on Nutritional Value and Edible Efficacy of Coix Seed. Grain Processing, 41, 50-52.

[3] Hui, R.H., Hou, D.Y., Guo, H., Liu, X.Y. and Li, X.C. (2005) Analysis of Nutritional Components in Coix Rice. Food Science, No. 8, 375.

[4] Pang, X.F., Zhang, S.W., Qu, Z.C., Jiang, B.K. and Liu, A.L. (1996) Growth Characteristics and High Yield Cultivation Techniques of Coix lacryma jobi. Shandong 
Agricultural Science, No. 3, 15-17.

[5] Zhao, X.H. (2002) Nutritional and Medical Value of Coix Rice and Development Prospect of Beverage Making. Shanxi Food Industry, No. 3, 35-36.

http://dx.chinadoi.cn/10.3969/j.issn.1673-6044.2002.03.015

[6] Gu, G.Y. (1990) Pharmacological Effect of Coix Seed. Chinese Traditional Patent Medicine, No. 12, 8-9.

[7] Yang, N.W. (2010) Formation and Regulating of Yield and Edible Quality of Two Coix lacryma jobi L. Peking Union Medical College and Chinese Academy of Medical Sciences, Beijing. http://dx.chinadoi.cn/10.7666/d.Y1775990

[8] Zhao, Y.J. and Liu, Z.H. (1992) Study on the Planting Density of Coix. Chinese Medicinal Materials, No. 12, 11-13.

[9] Zhang, Y.M., Yang, J.S., Zhao, Y.J. and Li, X.N. (2002) Advances in Chemical Constituents and Pharmacological Activities of Coix lacryma-jobi. Chinese Pharmaceutical Journal, 37, 8-11.

http://dx.chinadoi.cn/10.3321/j.issn:1001-2494.2002.01.004

[10] Agriculture-Cereal Foods (2020) Studies from Northeast Agricultural University Provide New Data on Cereal Foods [Understanding the Mechanism of Change In Morphological Structures, Visualization Features, and Physicochemical Characteristics of Adlay Seeds (Coix Lacryma-jobi)]. Food Weekly News.

[11] Yao, F.J. (2005) Effects of Photoperiod and Sowing Date on Growth and Yield of Coix. Sichuan Agricultural University, 16-19.

[12] Li, Z.F. and Liu, K. (2012) Properties of Job's Tears and High Yield Cultivation Techniques in Liaoning Province. Agricultural Science \& Technology and Equipment, No. 1, 66-69.

[13] Qian, M.X. (2016) Study on Suitable Row Spacing and Planting Density of Coix lacryma jobi. Peking Union Medical College and Chinese Academy of Medical Sciences, Beijing. http://dx.chinadoi.cn/10.7666/d.Y3075222

[14] Li, J.M. and Jin, Z.X. (2010) Potential Allelopathic Effects of Mikania Micrantha on the Seed Germination and Seedling Growth of Coix lacryma-jobi. Weed Biology and Management, 10, 194-201. https://doi.org/10.1111/j.1445-6664.2010.00384.x

[15] Zheng, X.L. (2014) Problems and Development Strategies of Coix Production in China. Agricultural Development and Equipments, No. 5, 40.

[16] Hu, J. and Jin, G.L. (2007) Nutritional and Medicinal Value of Coix Seeds. Food and Nutrition in China, No. 6, 57-58.

[17] Wu, Q.H., Wei, R.C. and Lin, W. (2014) Research Progress of Cultivation Technology of Coix lacryma-jobi. Research and Practice on Chinese Medicines, 28, 75-78.

[18] Song, Z.Q., Ao, M.H., Shen, G. and Liu, P.F. (2015) Effect of Planting Density, Fertilizer Level, and Number of Seedlings per Hole on Yield of Coix Lachryrma-jobi L. Asian Agricultural Research, 7, 69-73.

[19] Agostinho Ferreira, R., Borella, J., Moll Hüther, C., Barbosa do Canto, A.C., da Costa Correa, N.P., Marques Correia, D., Pereira Borges, R., de Pinho, C.F., de Barros Machado, T. and Rodrigues Pereira, C. (2020) Drought-Induced Stress in Leaves of Coix lacryma-jobi L. under Exogenous Application of Proline and GABA Amino Acids. Brazilian Journal of Botany, 43, 513-521. https://doi.org/10.1007/s40415-020-00637-0 